\title{
Propuesta de mejores prácticas: ITIL para la gestión de las TIC en apoyo a la actividad docente
}

\section{Proposal for best practices: ITIL in TIC management in support of teaching activity}

Blanca Faustina Hidalgo Ponce. ${ }^{1}$, Natalia Patricia Layedra Larrea. ${ }^{2}$ \& Marco Vinicio Ramos Valencia. $^{3}$

Recibido: 05-07-2019 / Revisado: 15-07-2019 /Aceptado: 12-08-2019/ Publicado: 10-09-2019

\begin{abstract}
DOl: https://doi.org/10.33262/cienciadigital.v3i3.4..844
\end{abstract}
This research proposes to guide teachers in the management of the use of TIC in the teaching-learning process, enabling the improvement in the quality of education and thus the ability to learn. Together with TIC, the integration of information management proposed in ITIL (Information Technology Infrastructure Library) has been considered. The ITIL philosophy adopts process management and considers that to achieve the key objectives of service management, processes should be used by people and tools effectively, efficiently and economically in the development of quality and innovation of information technology services aligned with business processes in this case to the teaching-learning process.

The hypothesis was: "A proposal that incorporates the best practices focused on the management, dissemination and use of TIC in the academic area will improve the quality of the teaching-learning process" using the chi-square test.

The proposed guide aims to offer precise guidance for those who intend to generate educational content using TIC

Keywords: TIC, ITIL, best practices, information technology, Quality of the information.

\section{Resumen}

Esta investigación propone guiar a los docentes en la gestión del uso de las TIC en el proceso de enseñanza aprendizaje, posibilitando la mejora en la calidad de la educación y con ello la capacidad de aprender. Junto a las TIC se ha considerado la integración

${ }^{1}$ Escuela Superior Politécnica de Chimborazo, Facultad de Informática y Electrónica. Riobamba, Ecuador. bhidalgo@espoch.edu.ec

2 Escuela Superior Politécnica de Chimborazo, Facultad de Mecánica. Riobamba, Ecuador. nlayedra@espoch.edu.ec

${ }^{3}$ Escuela Superior Politécnica de Chimborazo, Facultad de Informática y Electrónica. Riobamba, Ecuador. vi_ramos@espoch.edu.ec 
de la gestión de información propuestas en ITIL (Information Technology Infrastructure Library Librerías para la Infraestructura de las Tecnologías de la Información). La filosofía ITIL adopta la gestión de procesos y considera que para lograr los objetivos claves de la administración de servicios, los procesos deberían ser usados por las personas y las herramientas de manera efectiva, eficiente y económicamente en el desarrollo de la calidad y la innovación de los servicios de TI alineados con los procesos de negocio en este caso al proceso de enseñanza aprendizaje. Como hipótesis se planteó: "Una propuesta que incorpore las mejores prácticas enfocadas a la gestión, difusión y uso de las TIC en el área académica mejorará la calidad del proceso de enseñanza aprendizaje" utilizando la prueba de chi-cuadrado. La guía propuesta pretende ofrecer orientaciones mínimas para quienes se propongan generar contenidos educativos utilizando las TIC.

Palabras claves: Tecnologías de la información y comunicación, ITIL, mejores prácticas, tecnología de la información, calidad de la información.

\section{Introducción}

Este estudio se convierte en una propuesta de gestión en el uso de las TIC dentro de la ESPOCH (Escuela Superior Politécnica de Chimborazo), con el propósito de que sea una guía práctica y apoyo a la actividad docente para mejorar la calidad del proceso de enseñanza aprendizaje a los futuros profesionales mediante una formación basada en la correcta utilización de las TIC que faciliten el desenvolvimiento en su profesión.

En la actualidad las universidades no cuentan con políticas claras respecto a la capacitación y actualización de sus docentes en el área de informática y su utilización en la práctica educativa, dejando esta tarea, exclusivamente en sus manos. Debido a ello y a pesar de reconocer su utilidad, los docentes se resisten a la incorporación de las nuevas tecnologías en el aula. Por el otro lado, los alumnos manifiestan un alto interés en las nuevas tecnologías, frecuentemente poseen conocimientos previos respecto a su uso, provocando cierto desfase en la comunicación profesor-alumno perjudicial en el proceso de enseñanza-aprendizaje Para cumplir con dicho objetivo este trabajo recoge un conjunto de directrices denominadas mejores prácticas y tomadas en ITIL para la gestión de servicios de Tecnología de Información que guían al docente hacia la innovación y gestión de las herramientas que tiene a su alcance para mejorar la calidad del proceso de enseñanza aprendizaje.

Como resultado se propone una guía de fácil uso y aplicación para que los docentes desarrollen sus cátedras apoyados en las TIC buscando que los conceptos sean asimilados de la mejor manera como parte del conocimiento de los futuros profesionales.

\section{Antecedentes.}

La sociedad de la información(Fundación Telefónica, 2016, 2017) tiene como objetivo básico alcanzar el acceso universal a la información. Se fundamenta en una infraestructura que conecte en una sola red todos los servicios relacionados con la información ya sea a través de ordenadores, televisión, teléfono o cualquiera otro medio.

La "Sociedad o era de la Información se enfoca a la utilización masiva de herramientas electrónicas con fines de producción, intercambio y comunicación. Estas herramientas son conocidas como Tecnologías de la Información y las Comunicaciones (TIC)" (Varela, Pertejo, \& Martínez, 2005)y pueden clasificarse de la siguiente manera(Arcos Medina Gloria, 2005): 
- PC, servidores o cualquier otro dispositivo de hardware

- Telecomunicaciones

- Herramientas de productividad administrativas

- Productos inteligentes

\section{¿Qué es ITIL?}

ITIL como su nombre lo expresa es un grupo de libros, los cuales publican un conjunto de mejores prácticas para la gestión de servicios de Tecnología de Información, conocidas como ITSM(IT Service Management Gestión de servicios de TI)(Dabade, s. f.). El propósito de la gestión de servicios de TI es cerrar la brecha entre el negocio y la tecnología. En la figura 1 se presentan los libros que integran a ITIL.

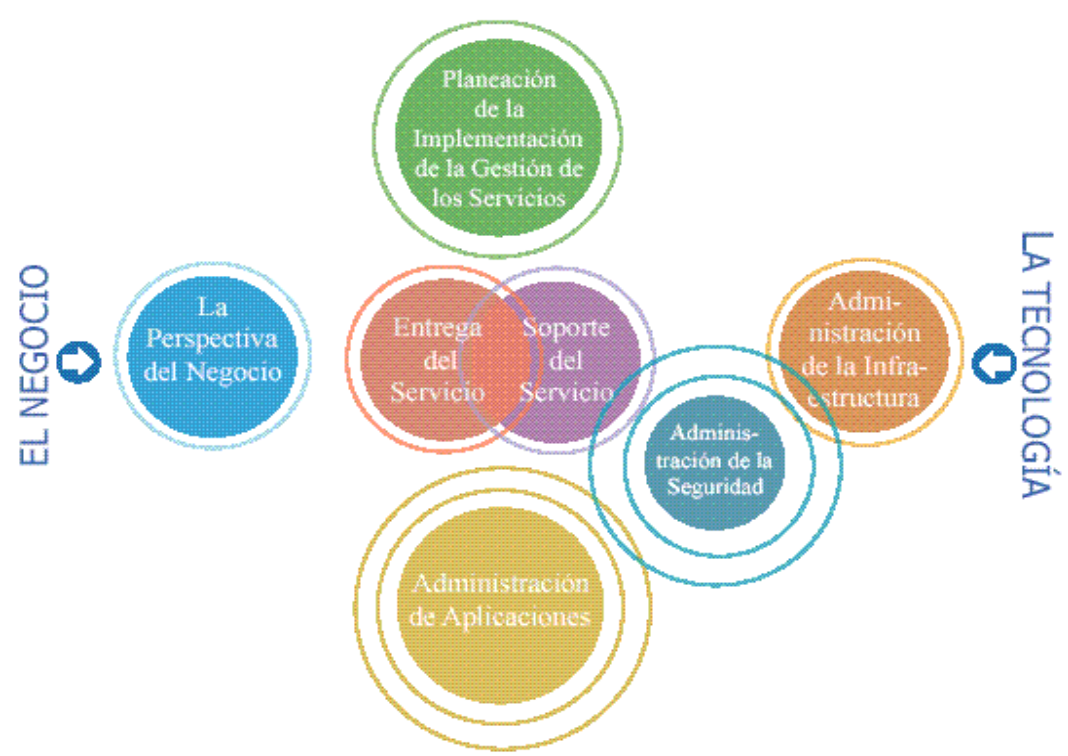

Figura 1: Estructura de ITIL

ITIL presenta las mejores prácticas de gestión de servicios de TI integradas bajo el enfoque de procesos(Bon \& De Jong, 2010), todos ellos orientados a brindar los servicios que el negocio requiere de $\mathrm{TI}$.

Beneficios generales de la implementación de ITIL Para el negocio

- Mejora de la calidad del servicio, traducida en un soporte más confiable para el negocio por parte del departamento de TI

- Mejora en la satisfacción del cliente ya que los proveedores de TI saben y entregan lo que se espera de ellos

- Mayor flexibilidad para el negocio a través de un entendimiento mejorado del soporte de TI 
Para el departamento de TI

- Contar con una visión clara de las capacidades reales de TI, aumentando la posibilidad de medirlas y por tanto de mejorarlas

- Incrementar el aprovechamiento de los recursos de TI

- Establecer mecanismos formales para el aprendizaje de experiencias previas

- Aumentar la motivación y satisfacción del personal respecto a su trabajo gracias al mejor entendimiento de sus capacidades y mejor manejo de sus expectativas

Las áreas cubiertas por ITIL(Guzmán, 2011), son:

- Soporte al servicio

- Entrega del servicio

- Planificación de la implantación

- Administración de aplicaciones

- Administración de la infraestructura de tecnologías de la información y comunicaciones

- Administración de seguridad

- Administración de activos de software

\section{Creación de materiales para la innovación educativa con TIC}

La introducción de tecnologías en los procesos de enseñanza aprendizaje puede ser una línea de formación permanente y de innovación educativa(Moguel Marín Susana Friné \& Alonzo Rivera Diana Lizbeth, 2009). Este proceso de innovación debería realizarse de forma escalonada, con apoyo desde las instituciones y dando un tiempo de interiorización.

Este proceso de innovación se hace realidad cuando se hace marcada diferencia entre, el uso de tecnologías sin cambiar los modelos tradicionales, por un lado, con el cambio de estos modelos de enseñanza con nuevas tecnologías, por otro lado. Esta última concepción nos lleva a un modelo centrado en el estudiante como eje de la enseñanza, y no el docente o los materiales didácticos como ha sucedido en los modelos más tradicionales(PricewaterhouseCoopers, 2017). En este nuevo modelo los docentes y los estudiantes toman una actitud más activa en el proceso de enseñanza y aprendiza con la utilización y producción de materiales didácticos.

El diseño y creación de materiales por docentes y estudiantes difiere por el nivel de producción, no obstante, y casi en todos los casos, en mayor o menor medida, realizan alguna adaptación de los materiales curriculares existentes o de los recursos disponibles en las distintas fuentes (televisión, vídeos, internet, etc). Estas etapas son interesantes analizarlas pues nos pueden permitir confeccionar un itinerario para planes de formación.

\section{Gestión de servicios de TI (Tecnologías de Información)}

La concienciación de que los servicios de TI son cada vez más importantes para el negocio ha llevado a la introducción de la gestión de servicios TI. La gestión de servicios TI (Guzmán, 2011)se dirige a proporcionar datos para la toma de decisiones desde una perspectiva de procesos, y proporcionar una implementación profesional con 
responsabilidades bien definidas. Una disposición incondicional tanto de dirección como de personal TI para enfocarse en el cliente y en el servicio.

Tres objetivos persiguen la gestión de servicios TI:

- Alinear los servicios TI con las necesidades futuras y actuales del negocio y sus clientes

- Mejorar la calidad de los servicios TI

- Reducir el costo de provisión de servicios a largo plazo

Además, la gestión de servicios TI para que sea efectiva y eficiente integra tres conceptos: personas, procesos y herramientas.

\section{Mejores prácticas}

Las mejores prácticas en la gestión de servicios de TI han sido incorporadas como uno de los elementos en ITIL desde 1989. Las mejores prácticas actualmente se encuentran integradas con el BSI(British Standards Institute de Estándares Británicos)(Pechuán Ignacio Gil, 2006).

En la figura 2 se resume las guías genéricas que debería soportar una organización para sus procesos y procedimientos internos.

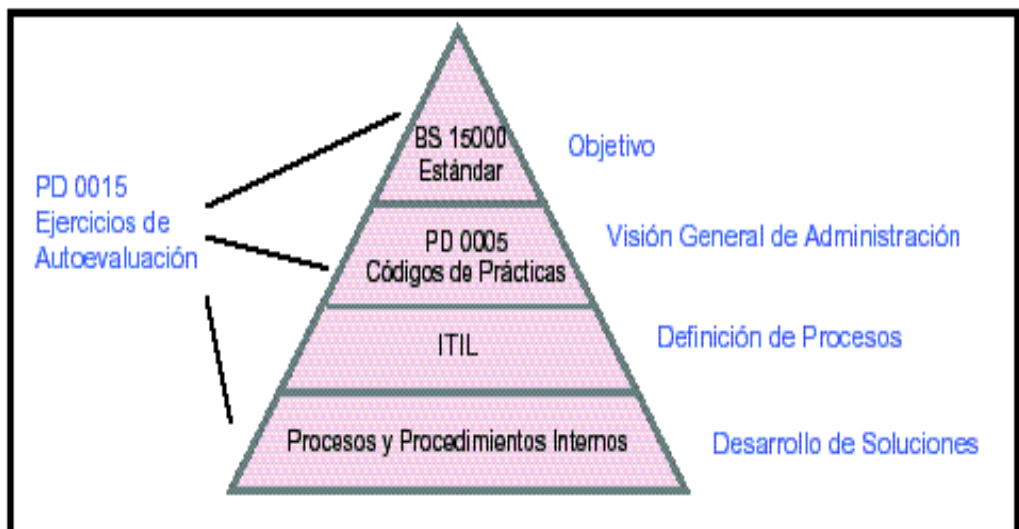

Figura 2: Guías de soporte ITIL para procesos y procedimientos internos

En la tabla 1 se definen el soporte de servicios según ITIL: 
Tabla 1. Soporte de servicios de ITIL

\begin{tabular}{|c|c|c|}
\hline $\begin{array}{c}\text { PROCESO O } \\
\text { FUNCIÓN DE ITIL }\end{array}$ & PROPÓSITO & PRINCIPALES BENEFICIOS \\
\hline Mesa de Ayuda & $\begin{array}{l}\text { Establecer un punto único } \\
\text { de contacto entre el } \\
\text { departamento de sistemas } \\
\text { y sus usuarios }\end{array}$ & $\begin{array}{l}\text { - Mejora en la administración y control de } \\
\text { las requisiciones de los usuarios al } \\
\text { departamento de TI } \\
\text { - Mejora de la comunicación y por tanto la } \\
\text { percepción de TI por parte del negocio }\end{array}$ \\
\hline $\begin{array}{l}\text { Administración } \\
\text { incidentes }\end{array}$ & $\begin{array}{l}\text { Restablecer lo antes } \\
\text { posible la operación } \\
\text { normal del servicio una } \\
\text { vez que se interrumpe }\end{array}$ & $\begin{array}{l}\text { - Resolución oportuna de interrupciones del } \\
\text { servicio de TI minimizando la afectación } \\
\text { del negocio } \\
\text { - Mejor organización de recursos } \\
\text { involucrados en restauración del servicio } \\
\text { de TI }\end{array}$ \\
\hline $\begin{array}{l}\text { Administración } \\
\text { problemas }\end{array}$ & $\begin{array}{l}\text { Resolver de raíz las } \\
\text { causas que interrumpen el } \\
\text { servicio }\end{array}$ & $\begin{array}{ll}\text { - } & \text { Disminución de interrupciones } \\
\text { recurrentes del servicio de TI al negocio } \\
\text { - } & \text { Mejora en la calidad de los servicios de TI }\end{array}$ \\
\hline $\begin{array}{l}\text { Administración } \\
\text { cambios }\end{array}$ & $\begin{array}{lcr}\text { Establecer } & \text { un } & \text { control } \\
\text { eficiente } & \text { sobre } & \text { los } \\
\text { cambios } & \text { de } & \text { la } \\
\text { infraestructura de TI } & \end{array}$ & $\begin{array}{l}\text { - Aseguramiento de que los cambios a la } \\
\text { infraestructura de TI son justificados y } \\
\text { aprobados } \\
\text { - Reducción de los riesgos en los servicios } \\
\text { de TI ocasionados por cambios en la } \\
\text { infraestructura }\end{array}$ \\
\hline $\begin{array}{l}\text { Administración } \\
\text { configuraciones }\end{array}$ & $\begin{array}{l}\text { Establecer mecanismos } \\
\text { estructurados de } \\
\text { liberación de hardware y } \\
\text { software }\end{array}$ & $\begin{array}{l}\text { - Alto nivel de casos exitosos en la } \\
\text { distribución y puesta en operación de } \\
\text { hardware y software para los usuarios de } \\
\text { TI } \\
\text { - Mejor administración y control de las } \\
\text { versiones de hardware y software } \\
\text { utilizados por los usuarios de TI }\end{array}$ \\
\hline
\end{tabular}

\section{Metodología}

El objetivo planteado de este trabajo es el de realizar una propuesta que recoja las mejores prácticas enfocadas a la gestión, y difusión sobre el uso de las nuevas tecnologías en el proceso enseñanza-aprendizaje en la educación superior con el fin de mejorar la calidad formativa de los futuros profesionales

En base a lo expuesto la hipótesis de esta definida de la siguiente manera:

$H i$ : Una propuesta que incorpore las mejores prácticas enfocadas a la gestión, difusión y uso de las TIC en el área académica de la ESPOCH mejorará la calidad del proceso de enseñanza aprendizaje.

\section{Universo y población}


La investigación aporta una serie de indicadores para evaluar la calidad del proceso de enseñanza aprendizaje con la utilización de las mejores prácticas enfocadas al uso de las TIC en la ESPOCH; el objeto de estudio, entre otras cosas, tiene como universo o población a los estudiantes matriculados en la institución en programas de formación presencial.

Como primer requisito para realizar esta investigación fue el de conformar el universo o población que debe tener las mismas características o propiedades, de aquí el carácter homogéneo de la misma.

Es importante indicar que para probar como influye el uso de TIC en el desarrollo de la práctica docente, se trabajó con grupos ya conformados por alumnos matriculados en la materia de Lenguajes de Programación I de la Escuela de Ingeniería en Telecomunicaciones y Redes.

\section{Procedimiento para la recolección de datos:}

La recolección de datos se efectuó de la forma siguiente:

- Se diseñó una guía de gestión y uso de las TIC en la docencia

- Identificación de los grupos ya conformados, se establece la validez y confiabilidad del instrumento a utilizar

- Se verificó la validez de la guía de gestión y uso de las TIC en la docencia y se concluyó que los instrumentos aplicados a la investigación son válidos y se adecua a sus objetivos. Se evaluaron, asimismo aspectos presentes en la teoría de TIC

- Se aplicaron los tratamientos pertinentes a cada grupo durante dos semanas, de acuerdo con la planificación establecida en el diseño instruccional de la asignatura y los planes de clase elaborados por la autora. Al grupo control (Paralelo "A") se impartió el contenido basado en estrategias de enseñanza tradicionales por parte de la investigadora; mientras al experimental (Paralelo "B") se aplicó el sistema utilizando las TIC diseñadas para su efecto, durante sesiones previamente fijadas, y con una duración establecida de acuerdo con sus intereses y ritmo de aprendizaje

- Para estas sesiones con el grupo experimental, el profesor participo exclusivamente como orientador del proceso de aprendizaje, las decisiones particulares sobre el mismo estuvieron en todo momento en manos de cada discente

- Finalizada la fase de los tratamientos, se procedió a desarrollar los test, los cuales fueron tabulados pertinentemente

- Para finalizar, se puso en práctica la técnica estadística seleccionada

\section{Resultados}

Con la finalidad de medir la influencia de la gestión y uso de las TIC en la calidad del proceso de enseñanza-aprendizaje se utilizó un cuestionario de preguntas cerradas con escala dicotómica y la técnica de la encuesta, definidas como un conjunto de preguntas respecto a una o más variables a medir, cuyas preguntas brindan a las personas las posibilidades de respuesta. Se desarrolló así una prueba aplicada posteriormente al tratamiento, cuya estructura fue la siguiente: Tres partes: La primera referida a la solicitud de la colaboración a los estudiantes; la segunda, a los datos académicos (experiencia con internet, etc.) de los estudiantes; y la tercera, al conjunto de ítems que conforman el instrumento. En cuanto a 
esta última parte, el instrumento estuvo constituido por veinticinco ítems en total, discriminados de acuerdo con los indicadores de las variables.

Cada uno de los indicadores presentó preguntas relacionadas con la totalidad de aspectos objeto de estudio.

\section{Procedimientos empleados:}

Con la finalidad de facilitar la comprensión procedimental, se dividió el estudio en procedimiento estadístico y procedimiento para la recolección de datos.

\section{Procedimiento estadístico:}

En primer término, se aplicó el Chi-cuadrado de Pearson, con esta prueba lo que queremos determinar es si la frecuencia observada de un fenómeno es significativamente igual a la frecuencia teórica prevista, o sí, por el contrario, estas dos frecuencias acusan una diferencia significativa.

\section{Resultados}

A continuación, se demuestra la tabla 2 como resumen del tratamiento de los datos con la operacionalización de las variables definidas en la hipótesis.

Tabla 2. Estadístico de los resultados de la investigación

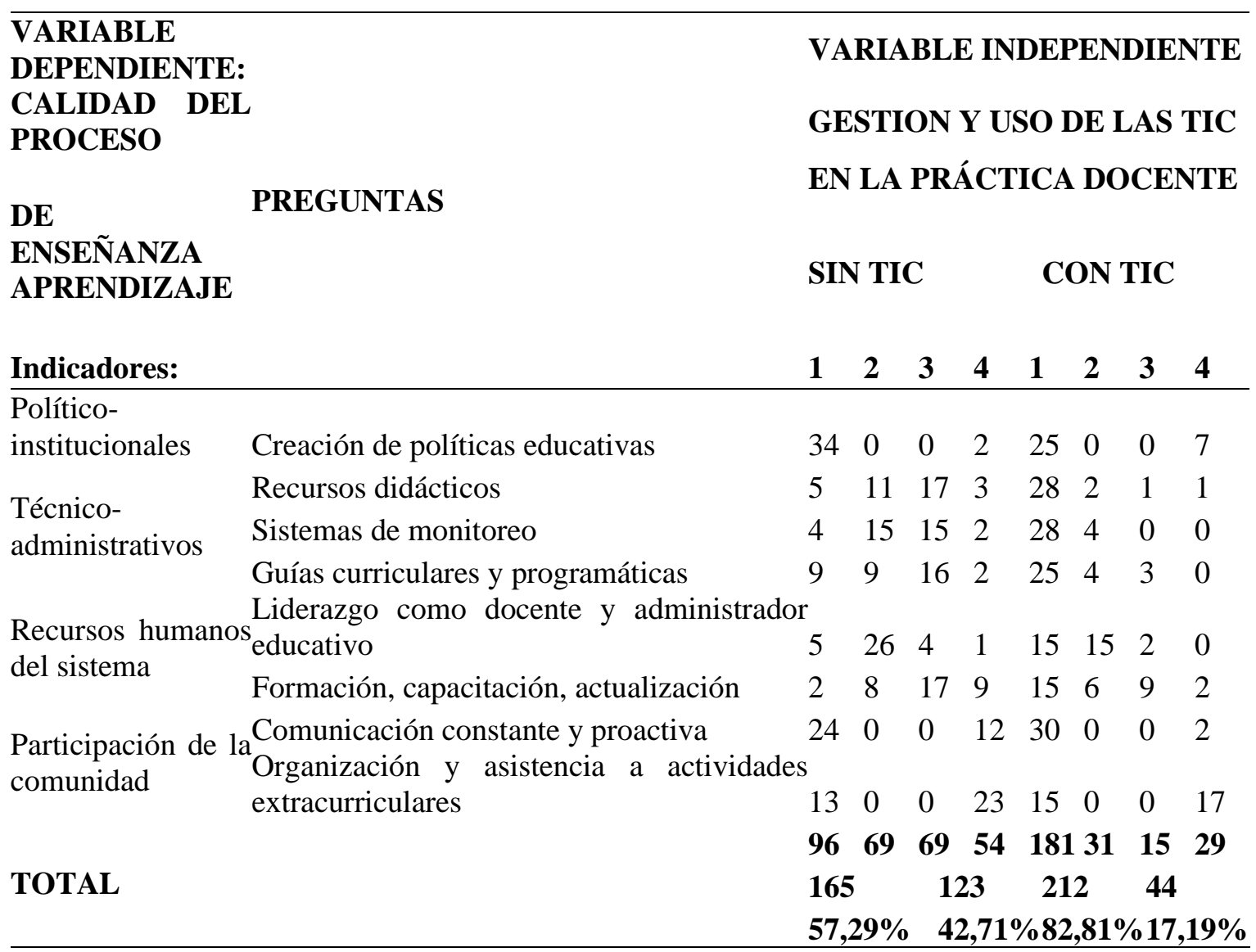


En la prueba del chi-cuadrado es necesario conformar una tabla de contingencias en la que se muestre las observaciones en cada una de las variables que conforman la hipótesis, la tabla 3 muestra dicha relación de la siguiente manera:

Tabla 3. Contingencia de las frecuencias observadas

\begin{tabular}{|c|c|c|c|}
\hline \multirow{2}{*}{$\begin{array}{c}\text { CALIDAD DEL PROCESO } \\
\text { DE ENSEÑANZA APRENDIZAJE }\end{array}$} & \multicolumn{3}{|c|}{$\begin{array}{l}\text { USO Y GESTION DE LAS TIC } \\
\text { EN LA PRÁCTICA DOCENTE }\end{array}$} \\
\hline & SIN TIC & CON TIC & TOTAL \\
\hline Mejora el proceso de enseñanza aprendizaje & 165 & 212 & 37 \\
\hline No mejora el proceso de enseñanza aprendizaj & 123 & 44 & \\
\hline TOTAL & 288 & 256 & \\
\hline
\end{tabular}

En este tipo de prueba a más de las frecuencias observadas, es necesario mostrar las frecuencias esperadas cuyos valores serían los que encontraríamos si no habría relaciones entre las variables.

Para calcular las frecuencias esperadas se aplican las fórmulas de Chi- cuadrado, y como resultado obtenemos la tabla 4:

Tabla 4. Frecuencias esperadas

\begin{tabular}{|c|c|c|c|}
\hline \multirow{2}{*}{$\begin{array}{c}\text { CALIDAD DEL PROCESO } \\
\text { DE ENSENAANZA APRENDIZAJE }\end{array}$} & \multicolumn{3}{|c|}{$\begin{array}{l}\text { USO Y GESTION DE LAS TIC } \\
\text { EN LA PRÁCTICA DOCENTE }\end{array}$} \\
\hline & SIN TIC & CON TIC & TOTAL \\
\hline Mejora el proceso de enseñanza aprendizaje & 199,59 & 177,41 & 377 \\
\hline No mejora el proceso de enseñanza aprendizaje & 88,41 & 78,59 & 167 \\
\hline TOTAL & 288 & 256 & 544 \\
\hline
\end{tabular}

Con el cálculo de las frecuencias tanto esperada como las observadas se aplica la formula siguiente:

$$
\chi^{2}=\sum_{i=1}^{r} \sum_{j=1}^{k} \frac{\left(O_{i j}-E_{i j}\right)^{2}}{E_{i j}}
$$

Donde:

- $\boldsymbol{O}_{i j}$ denota a las frecuencias observadas. Es el número de casos observados clasificados en la fila $\mathrm{i}$ de la columna $\mathrm{j}$

- $\quad \boldsymbol{E} \boldsymbol{i j}$ denota a las frecuencias esperadas o teóricas. Es el número de casos esperados correspondientes a cada fila y columna. Se puede definir como aquella frecuencia que se observaría si ambas variables fuesen independientes 
Tabla 5. Tabla del chi-cuadrado

\begin{tabular}{lccccc}
\hline CELDA & $\mathrm{O}$ & $\mathrm{E}$ & $\mathrm{O}-\mathrm{E}$ & $(\mathrm{O}-\mathrm{E})^{2}$ & $(\mathrm{O}-\mathrm{E})^{2} / \mathrm{E}$ \\
\hline Mejora/ sin TIC & 165,00 & 199,59 & $-34,59$ & 1196,35 & 5,99 \\
Mejora/ con TIC & 212,00 & 177,41 & 34,59 & 1196,35 & 6,74 \\
No mejora/ sin TIC & 123,00 & 88,41 & 34,59 & 1196,35 & 13,53 \\
No mejora/ con TIC & 44,00 & 78,59 & $-34,59$ & 1196,35 & 15,22 \\
& & & & $\mathrm{X}^{2}$ & 41,49 \\
\hline
\end{tabular}

Para completar la comprobación de la hipótesis, el método prevé determinar los grados de libertar mediante la fórmula:

$$
\mathrm{Gl}=(\mathrm{r}-1)(\mathrm{c}-1)
$$

Donde

r: es el número de filas de la tabla de contingencia

c: es el número de columnas de la tabla de contingencia

En este caso $\mathrm{Gl}=(2-1)(2-1)=1$

\section{Resultado}

De acuerdo a la tabla de valores del chi-cuadrado y a los niveles de confianza y el grado de libertad de 1. Calculamos el valor del punto crítico de una chi-cuadrado con un valor de a $=0,05$ (nivel de significación) y grados de libertad $\mathrm{Gl}=1$, es decir, $\mathrm{X}_{0,05}^{2}(1)=3.841$.

La prueba arroja un valor de $\mathrm{X}^{2}=41.49$, la región crítica es mayor o igual que 3.841, es decir, el intervalo (3.841 +infinito).

Como el valor del estadístico $\mathrm{X}^{2}=41.49$ es mayor que el punto crítico se rechaza la hipótesis nula Ho y por tanto se acepta la hipótesis de la investigación Hi:

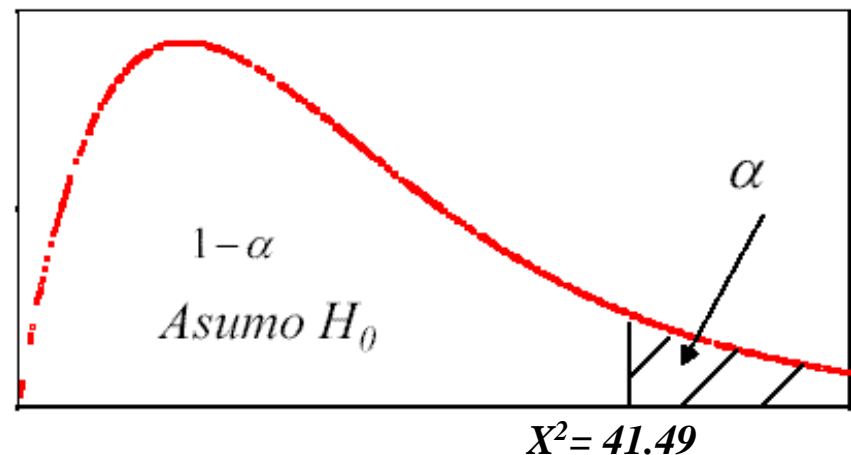

Figura 3: Distribución del Chi-cuadrado 


\section{Conclusiones}

- Las TIC aportan a la educación una nueva dimensión, las posibilidades de compartir, de transferir información y conocimientos básicos, facilitan el acceder a nuevas fuentes del saber, posibilitando la mejora de nuestra capacidad de aprender. Las nuevas tecnologías no sólo provocan el cambio del paradigma educativo, sino que además lo posibilitan, lo facilitan y ofrecen una dimensión nueva a lo esencial, es decir, al propio proceso de aprendizaje.

- El empleo de ITIL ayuda en la adopción de prácticas y procesos que permitirá optimizar las operaciones de TI. Además, que los responsables de las áreas funcionales tengan una mayor visibilidad sobre los procesos de TI. La adopción de ITIL junto con estándares de control y madurez de gestión en TI y con estándares para establecimiento de procesos de negocio y toma de decisiones permite medir el rendimiento de la organización de TI, resultando más sencillo para la dirección establecer objetivos y tomas de decisiones.

- Si se utiliza adecuadamente las TIC con un enfoque de gestión de ITIL existe una mejora cuantitativa del $25.5 \%$ del proceso de enseñanza aprendizaje ya que dichas tecnologías poseen cualidades que apoyan a: creación de políticas educativas, uso de recursos didácticos, desarrolla nuevos sistemas de evaluación y monitoreo de la práctica docente, y sobre todo facilitan la comunicación proactiva y constante con los involucrados en el proceso.

\section{Referencias bibliográficas}

- Arcos Medina Gloria. (2005). Integración de la Ingeniería de Software en la Creación de un Ambiente Colaborativo de Apoyo a la Educación Superior (ESPOCH). Recuperado de http://bibliotecas.espoch.edu.ec/cgi-bin/koha/opacdetail.pl?biblionumber $=41750 \&$ shelfbrowse_itemnumber $=60380$

- Bon, J. V., \& De Jong, A. (2010). Fundamentos de ITIL V3 (Tercera Edición). Van Haren.

- Dabade, T. D. (s. f.). NFORMATION TECHNOLOGY INFRASTRUCTURE LIBRARY (ITIL). Proceedings of the 4th National Conference; INDIACom-2010, 2. Recuperado http://www.bvicam.ac.in/news/INDIACom\%202010\%20Proceedings/papers/Group 3/INDIACom10_11_Paper\%20(4).pdf

- Fundación Telefónica, 2016. (2017). La Sociedad de la Información en España 2016 (N.o 36; p. 281). Recuperado de Telefónica website: https://www.google.com/url?sa=t\&rct=j\&q=\&esrc=s\&source=web\&cd=1\&cad=rja \&uact $=8 \&$ ved=2ahUKEwj_yo7f8-

TiAhWiTN8KHdMDBpwQFjAAegQIABAC\&url=https\%3A\%2F\%2Fpubliadmin.f undaciontelefonica.com\%2Findex.php $\% 2$ Fpublicaciones $\% 2$ Fadd_descargas $\% 3$ Ftip o_fichero\%3Dpdf\%26idioma_fichero\%3Des_es $\% 26$ title\%3DLa\%2BSociedad $\% 2 \mathrm{~B}$ de $\% 2 \mathrm{Bla} \% 2 \mathrm{BInformaci} \% 25 \mathrm{C} 3 \% 25 \mathrm{~B} 3 \mathrm{n} \% 2 \mathrm{Ben} \% 2 \mathrm{BEspa} \% 25 \mathrm{C} 3 \% 25 \mathrm{~B} 1 \mathrm{a} \% 2 \mathrm{~B} 2016$ $\% 26$ code $\% 3 D 558 \% 261$ ang\%3Des\%26file\%3DLa_Sociedad_de_la_Informacion_en _Espana_2016.pdf\&usg=AOvVaw1MYP6pnsefGVi3rTMDPVzS

- Guzmán, Á. (2011). ITIL v3 - Gestión de Servicios de TI - Dialnet. Recuperado 17 $\begin{array}{llll}\text { de } & \text { septiembre de }\end{array}$ https://dialnet.unirioja.es/servlet/articulo?codigo $=4001967$ 
- Moguel Marín Susana Friné, \& Alonzo Rivera Diana Lizbeth. (2009, junio 1). DIMENSIONES DEL APRENDIZAJE Y EL USO DE LAS TIC 'S. EL CASO DE LA UNIVERSIDAD AUTÓNOMA DE CAMPECHE, MÉXICO. RIED. Revista Iberoamericana de Educación a Distancia, 195-210. Recuperado de http://www.redalyc.org/pdf/3314/331427210010.pdf

- Pechuán Ignacio Gil. (2006). Sistemas y Tecnologías de la Información para la gestión. McGraw-Hill.

- PricewaterhouseCoopers. (2017, abril 9). Una mirada al futuro ¿Cómo cambiará el orden económico mundial para el 2050? Recuperado de https://www.pwc.com/co/es/assets/document/el_mundo_en_2050.pdf

- Varela, M. C., Pertejo, P., \& Martínez, I. M. P. (2005). Nuevas tecnologías en Lingüística, Traducción y Enseñanza de lenguas. Univ Santiago de Compostela (Vol. 1). Santiago de Compostela: Universidad Santiago de Compostela.

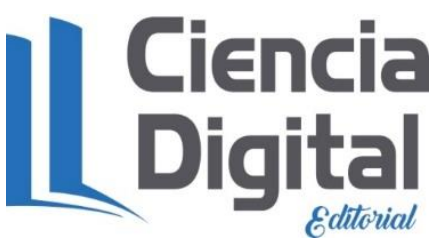




\section{PARA CITAR EL ARTÍCULO INDEXADO.}

Hidalgo Ponce, B., Layedra Larrea, N., \& Ramos Valencia, M. (2019). Propuesta de mejores prácticas: ITIL para la gestión de las TIC en apoyo a la actividad docente. Ciencia Digital, 3(3.4.), 167-179. https://doi.org/10.33262/cienciadigital.v3i3.4.844

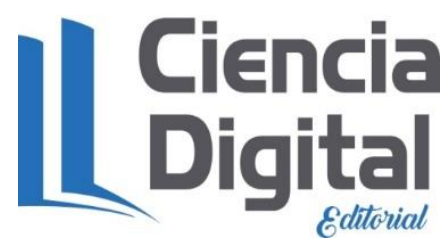

El artículo que se publica es de exclusiva responsabilidad de los autores y no necesariamente reflejan el pensamiento de la Revista Ciencia Digital.

El artículo queda en propiedad de la revista y, por tanto, su publicación parcial y/o total en otro medio tiene que ser autorizado por el director de la Revista Ciencia Digital.
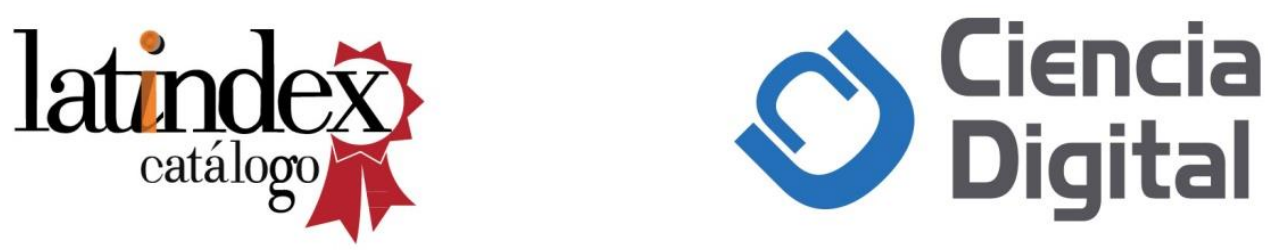